\title{
Search for gamma-ray lines in the Galaxy with DAMPE
}

\section{XU Zun-Lei, ${ }^{a}, b, *$ SHEN Zhao-Qiang, ${ }^{a, *}$ DUAN Kai-Kai, ${ }^{a}$ LI Xiang $^{a}$ and M. N. Mazziotta ${ }^{c}$ on behalf of the DAMPE Collaboration \\ (a complete list of authors can be found at the end of the proceedings)}

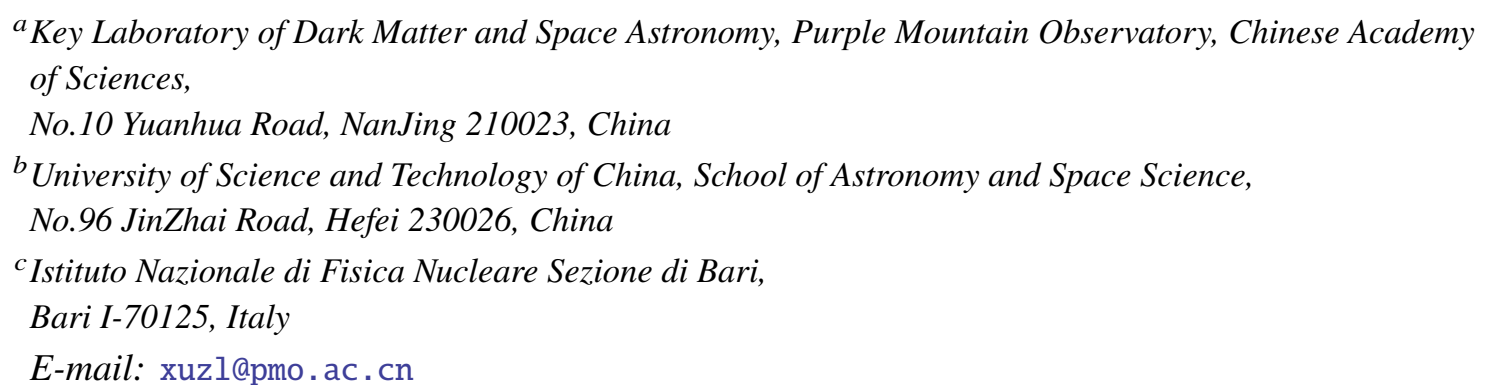

DArk Matter Particle Explorer (DAMPE) has a great potential in the search of monochromatic and sharp gamma-ray structures in $\mathrm{GeV}-\mathrm{TeV}$ range thanks to its good energy resolution. In this work, we search for gamma-ray line structures using 5.0 years of DAMPE data. To improve the sensitivity, we develop two types of data sets and adopt the signal-to-noise ratio optimized regions of interest (ROIs) for different DM density profiles. No line signals or candidates, including those located at $133 \mathrm{GeV}$ and $43 \mathrm{GeV}$, are found between $10 \mathrm{GeV}$ and $300 \mathrm{GeV}$ in the Galaxy. Therefore we calculate the $95 \%$ confidence level constraints on the velocity-averaged cross section for $\chi \chi \rightarrow \gamma \gamma$ and the decay lifetime for $\chi \rightarrow \gamma v$ with systematic uncertainties included. Our constraints on DM parameters are mostly comparable to the Fermi-LAT 5.8-yr results. The lower limit for DM decay lifetime below $100 \mathrm{GeV}$ are better than that of Fermi-LAT.

$37^{\text {th }}$ International Cosmic Ray Conference (ICRC 2021)

July 12th - 23rd, 2021

Online - Berlin, Germany

\footnotetext{
${ }^{*}$ Presenter
} 


\section{Introduction}

According to the recent observation of cosmic microwave background, the current mean energy density of matter is around 6.4 times that of baryons [1].The discrepancy is usually explained with an extra cold matter component - the dark matter (DM). Weakly interacting massive particle (WIMP) is a favorable DM candidate, since it provides a natural explanation of the DM relic density [2]. And peaked spectral features may also arise due to the virtual internal bremsstrahlung process in the DM annihilation [3] or the decay of low-mass intermediate particles generated by the annihilating or decaying DM [4]. Since the line-like spectrum is hard to be produced in known astrophysical processes, a robust detection would be a smoking-gun signature of WIMPs. Many works have been carried out on gamma-ray line search using the Fermi-LAT data, but only some tentative signals with low confidence are found so far [5]. Given the high energy resolution of DAMPE among the detectors of similar kind, it has a unique advantage in the search of a gamma-ray line signal.

The number of photons in a line signal is proportional to the acceptance of the instrument, while the number of background photons underlying the line structure is proportional to the product of the acceptance and the energy dispersion width. To improve the sensitivity, the key issue is to improve the acceptance and energy resolution of the detector. In section 2, We quantitatively prove that the sensitivity of gamma-ray line signal search is positively correlated with the ratio of acceptance to energy resolution. We have developed a standard gamma-ray data sample before [6,7], which, however, is not optimized for line search. In order to maximize the sensitivity of gamma-ray line search, it is necessary to balance the acceptance and energy resolution in the photon selection algorithm. We therefore developed a photon sample specifically optimized for line search, and this line-search sample is described in detail in section 3. On the other hand, the increase of photon statistics can significantly improve the sensitivity of line search. For this purpose, we also find in this work the photons that convert into $e^{+} e^{-}$pairs in the BGO calorimeter. With only BGO track but no STK track, these photons of poor angular resolution are but the statistics can be increased. The selection of these BGO-only photons is also introduced. And at the end, we perform a line search using the latest DAMPE gamma-ray observation of inner Galaxy and then derive the constraints on the DM parameters.

\section{Sensitivity for linelike structures}

We use the ratio of acceptance $(\mathcal{A})$ to the half width of $68 \%$ energy containment $(\Delta E / E)$ as the optimized target for the line-search sample, since this quantity is positively correlated to the signal-to-noise ratio of lines. Qualitatively, the local significance of a line structure can be written as $n_{\text {line }} / \sqrt{n_{\text {bkg,eff }}}$, where $n_{\text {line }}$ and $n_{\text {bkg,eff }}$ represent the photon counts from the line and from the background emission, respectively. The line counts from given targets are proportional to the acceptance and the observing time $T$, and the $T$ is related to the field of view proportionally when the satellite orbit is given, therefore we have $n_{\text {line }} \propto \mathcal{A}$. Concerning the background counts, since only the background fluctuation under the line can directly expose or hide a signal, we use the counts of background emission under the line peak rather than the counts in the entire energy range as $n_{\mathrm{bkg} \text {,eff }}$, i.e. the "effective background" in previous works $[8,9]$. The effective background counts can be approximated using the counts integrated around the line energy $E$, 


$$
\begin{aligned}
n_{\mathrm{bkg}, \mathrm{eff}} & \approx \int_{E-\Delta E}^{E+\Delta E} F_{\mathrm{bkg}}\left(E^{\prime}\right) \epsilon\left(E^{\prime}\right) T \mathrm{~d} E^{\prime} \\
& \approx F_{\mathrm{bkg}}(E) \epsilon(E) T \cdot 2 \Delta E,
\end{aligned}
$$

where $F_{\mathrm{bkg}}(E)$ is the spectrum of background emission, and $\Delta E$ is the half width of the line. Based on the same argument above, $n_{\mathrm{bkg}, \mathrm{eff}} \propto \mathcal{A} \times \Delta E / E$. Therefore the significance of a line improves when the quantity $\sqrt{\mathcal{A} /(\Delta E / E)}$ increases. On the other hand, when no line signal exists, the $95 \%$ confidence level upper limit of the counts is $1.64 \sigma$ deviated from the null model, so we have $n_{\text {line,UL }}=1.64 \sqrt{n_{\text {bkg,eff }}}$. The upper limit on the line spectrum is $F_{\text {line, UL }}=n_{\text {line,UL }} /(\epsilon T) \delta(E-$ $\left.E_{\text {line }}\right) \propto \sqrt{(\Delta E / E) / \mathcal{A}}$.

\section{Data selection}

In the last section, we show that a larger ratio $(R)$ between the acceptance and the energy resolution is required to improve the sensitivity of gamma-ray line search. In practice, however, there is a trade-off between these two quantities. A smaller $Z$ value in the BGO through which the particle track is required to pass leads to a larger field of view, which improves the acceptance. On the other hand, a smaller $Z$ value also means that particles leak more energy outside of the BGO detector, resulting in a poor energy resolution. And vice versa if a larger $Z$ value is required. We thus need to find an optimal $Z$ value to maximize the $R$ value.

We simulate the relationship between the $R$ value and the $Z$ value at different energies. And we use the polynomial $\frac{Z}{\mathrm{~mm}}=97.78+102.17 \times \frac{E}{\mathrm{GeV}}-11.96 \times\left(\frac{E}{\mathrm{GeV}}\right)^{2}$ to fit the $Z$ value corresponding to the maximum $R$ in different energy, and we can get the analytical relationship between the optimal $Z$ value and energy in different energies.As shown in Fig. 1, we use the fitted optimal $Z$ value to get the $R$ curves of different energies. We can see that the $R$ value obtained by this method is better than a fixed $Z$ value.

Only about $50 \%$ of the photons have electron pair conversion in the STK, the rest about $40 \%$ of the photons have electron pair conversion in the BGO. With only BGO track but no STK track, these photons have poorer direction measurements. The search of line requires as many photons as possible with high energy resolution, and the angular resolution is not a critical issue here, so it is necessary to count in the photons with only BGO track. The selection process of the photons with only BGO track is similar to that of the photons with STK track. Firstly, most protons are rejected according to the shower morphology of the incident particles in the BGO calorimeter, and then the BGO track is reconstructed. Finally, PSD is used as an anti coincidence detector to distinguish photons from charged particles. The challenge is that if the reconstructed track deviates greatly from the original one, a wrong PSD crystal will be found and a background event maybe introduced. The BGO track reconstruction is based on the centroid method. More accurate centroids determined on more layers result into a track of higher quality. Here we choose the photons passing through more than 8 layers of BGO.

As shown in Fig. 2, at present, the acceptance of photons with only BGO track is only about one tenth of that of the photons with STK track (in the low-energy), which is consistent with the ratio of the number of cases between the two data. The reason of the small acceptance of the photons with 


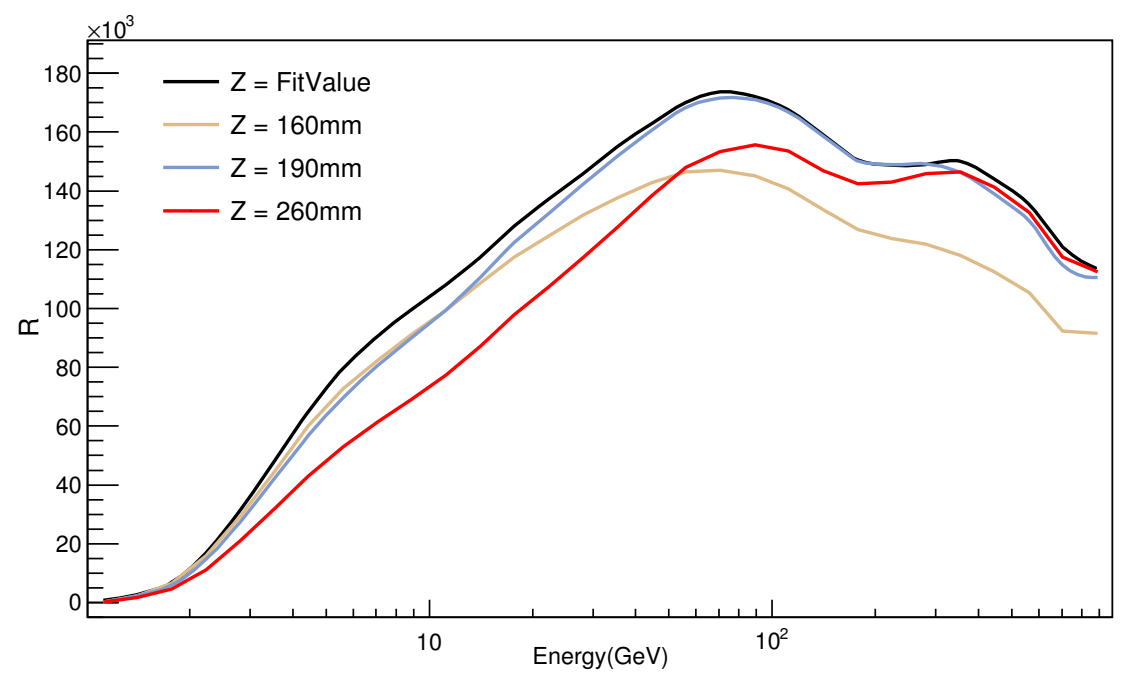

Figure 1: The $R$ value at different $Z$ values and energy.



Figure 2: The acceptance of photons with only BGO track and the photons with STK track.

only BGO track is that the electron pair conversion of these photons occurs in the BGO, and thus the energy deposited in the first layer of BGO is less, which leads to a lower trigger efficiency [10].

\section{Line search in the Galactic center}

DM density profile $\rho_{\mathrm{DM}}$ is uncertain particularly in the inner Galaxy, so we consider three reference profiles: the Navarro-Frenk-White (NFW) profile $\rho_{\mathrm{NFW}}(r)=\rho_{s} /\left[\left(r / r_{s}\right)\left(1+r / r_{s}\right)^{2}\right]$ with $r_{s}=20 \mathrm{kpc}$ [11]; the Einasto profile $\rho_{\text {Ein }}(r)=\rho_{s} \exp \left\{-(2 / \alpha)\left[\left(r / r_{s}\right)^{\alpha}-1\right]\right\}$ with $r_{s}=20 \mathrm{kpc}$ and $\alpha=0.17$ [12, 13]; the isothermal profile $\rho_{\text {iso }}(r)=\rho_{s} /\left[1+\left(r / r_{s}\right)^{2}\right]$ with $r_{s}=5 \mathrm{kpc}$ [14]. The normalization $\rho_{s}$ can be found with $\rho_{\mathrm{DM}}\left(R_{0}\right)=0.4 \mathrm{GeV} \mathrm{cm}^{-3}$ and $R_{0}=8.5 \mathrm{kpc}$. 
For either the annihilating or decaying DM, we make regions of interest (ROIs) optimized for the sensitivity considering different DM density profiles. The ROIs are circular regions with radius $R_{\mathrm{GC}}$ centering at the Galactic center but with the rectangular region $|l| \geq \Delta l$ and $|b| \leq 5$ deg masked. For the annihilating DM, the optimal $\left(R_{\mathrm{GC}}, \Delta l\right)$ is $(86 \mathrm{deg}, 0 \mathrm{deg})$ for the isothermal profiles. For the decaying DM, all the optimal $\left(R_{\mathrm{GC}}, \Delta l\right)$ for different profiles are close to $(150 \mathrm{deg}, 0 \mathrm{deg})$, so this parameter set is adopted as a representative.

We perform an unbinned likelihood analysis with the sliding windows technique to quantify the significance of the hypothesized lines, which will mitigate the bias caused by the background spectral shape and energy binning. For a line at $E_{\text {line }}$, only the photons in the window from $0.5 E_{\text {line }}$ to $1.5 E_{\text {line }}$ are used in the fit. The energy difference between two adjacent lines is $0.5 \sigma_{E}$, where $\sigma_{E}$ is the half width of the $68 \%$ exposure weighted energy dispersion containment in the Galactic center for LineSearch data set $[15,16]$. The unbinned likelihood function $L_{k}(\Theta)$ for the data set $k$ in the energy window of $\left[E_{\min }, E_{\max }\right]$ is defined as

$$
\ln L_{k}(\Theta)=\sum_{i=1}^{n_{k}} \ln \left[\bar{\lambda}_{k}\left(E_{i} ; \Theta\right)\right]-\int_{E_{\min }}^{E_{\max }} \bar{\lambda}_{k}(E ; \Theta) \mathrm{d} E .
$$

$n_{k}$ is the number of observed photons in the given energy range, $\bar{\lambda}_{k}(E ; \Theta)$ is the expected counts in model per energy range with the parameter $\Theta$, which is calculated with the exposure $\bar{\epsilon}_{k}(E)$ at energy $E$ averaged over the ROI. The likelihood to be fitted is $L(\Theta)=L_{1}(\Theta) \times L_{2}(\Theta)$, where the subscript indices represent two data sets.

In each energy window, a likelihood ratio test [17] is performed. The null hypothesis consists of a power-law background, i.e. $\bar{\lambda}_{\text {null, } k}(E ; \Theta)=F_{\mathrm{b}}(E) \bar{\epsilon}_{k}(E)$, while the signal hypothesis contains a monochromatic line and a power-law background, i.e. $\bar{\lambda}_{\mathrm{sig}, k}(E)=F_{\mathrm{b}}(E) \bar{\epsilon}_{k}(E)+$ $\bar{F}_{\mathrm{s}, k}(E) \bar{\epsilon}_{k}\left(E_{\text {line }}\right)$. The power-law spectrum and the line structure are defined as $F_{\mathrm{b}}\left(E ; N_{\mathrm{b}}, \Gamma\right)=$ $N_{\mathrm{b}} E^{-\Gamma}$ and $\bar{F}_{\mathrm{s}, k}\left(E ; N_{\mathrm{s}}, E_{\text {line }}\right)=N_{\mathrm{s}} \bar{D}_{\text {eff }, k}\left(E ; E_{\text {line }}\right)$ (i.e. $S_{\text {line }}(E)=N_{\mathrm{s}} \delta\left(E-E_{\text {line }}\right)$ before convolution) respectively. $\bar{D}_{\text {eff }}$ is the exposure weighted energy dispersion function averaged over the ROI and is given by

$$
\bar{D}_{\mathrm{eff}, k}\left(E ; E_{\text {line }}\right)=\frac{\sum_{i j} D_{k}\left(E ; E_{\text {line }}, \theta_{j}\right) \epsilon_{k}\left(E_{\text {line }}, \theta_{j}, \mathbf{r}_{i}\right)}{\sum_{i j} \epsilon_{k}\left(E_{\text {line }}, \theta_{j}, \mathbf{r}_{i}\right)}
$$

where $\theta$ is the incident angle with respect to the boresight, $\mathbf{r}$ is the pixel coordinate within the ROI, and $D(E)$ is the energy dispersion function of DAMPE [7]. We fit both the models to the data using the MINUIT [18] and then calculate the test statistic (TS) value TS $\equiv-2 \ln \left(\hat{L}_{\text {null }} / \hat{L}_{\text {sig }}\right)$, where $\hat{L}_{\text {null }}$ and $\hat{L}_{\text {sig }}$ are the maximum likelihood values of the null and alternative model respectively.

We do not find any gamma-ray line signal or candidate (TS $\geq 9$ ) in all the ROIs (Fig. 3), including the $133 \mathrm{GeV}$ and $43 \mathrm{GeV}$ candidates suggested in the Fermi-LAT data. Therefore we calculate the $95 \%$ confidence level constraints on the DM parameter space.

For annihilating DM, the gamma-ray spectrum is given by

$$
S_{\text {line }}(E)=\frac{1}{4 \pi} \frac{J_{\mathrm{DM}} \times\langle\sigma v\rangle_{\gamma \gamma}}{2 m_{\chi}^{2}} 2 \delta\left(E-E_{\text {line }}\right),
$$

where $\langle\sigma v\rangle_{\gamma \gamma}$ is the velocity-averaged annihilation cross section for $\chi \chi \rightarrow \gamma \gamma$, and $m_{\chi}$ is the rest mass of a DM particle which satisfies $E_{\text {line }}=m_{\chi} c^{2}$. For the decaying DM, the spectrum can be 


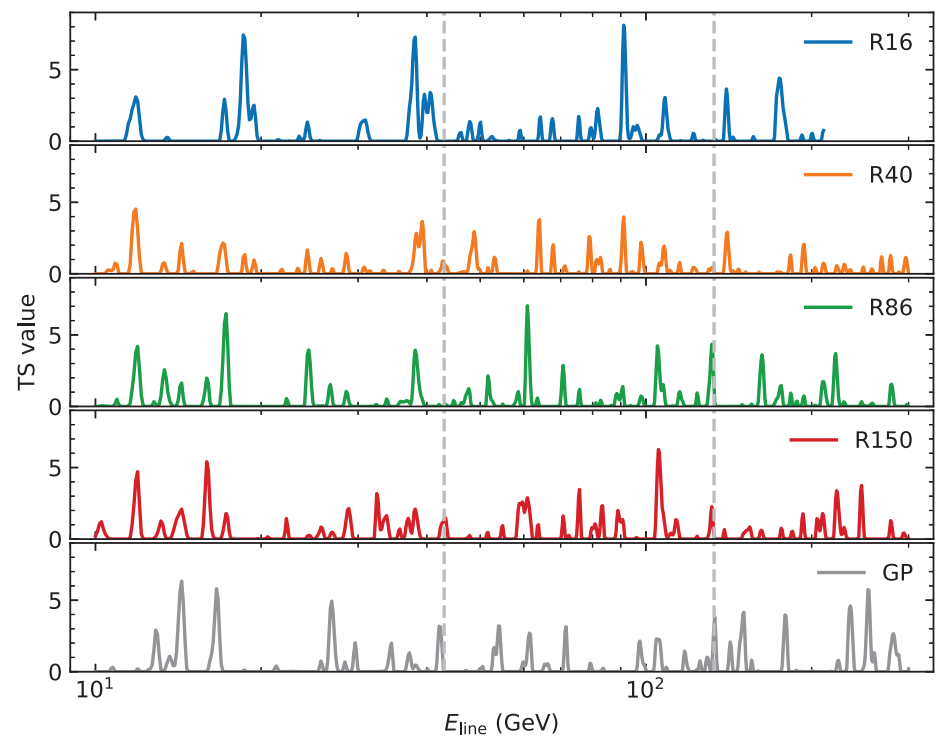

Figure 3: TS values of line candidates at various energies in the signal optimized ROIs and the Galactic plane region $(|l|>30 \mathrm{deg}$ and $|b|<5 \mathrm{deg})$. The local significance can be calculated with $s_{\text {local }}=\sqrt{\mathrm{TS}}[8]$. Dashed gray lines correspond to the line candidates at $43 \mathrm{GeV} \mathrm{[16]} \mathrm{and} 133 \mathrm{GeV}$ [19]. The highest line energy for R16 ROI is $211 \mathrm{GeV}$ which is limited by the minimum photon counts we required in a window.

written as

$$
S_{\text {line }}(E)=\frac{1}{4 \pi} \frac{D_{\mathrm{DM}}}{m_{\chi} \tau_{\gamma \nu}} \delta\left(E-E_{\text {line }}\right),
$$

where $\tau_{\gamma \nu}$ is the particle lifetime of a DM particle through the $\chi \rightarrow \gamma v$ process and $E_{\text {line }}=0.5 m_{\chi} c^{2}$. We increase (decrease) the cross section (lifetime) from its best-fit value until the log-likelihood changes by 1.35 to achieve the constraints.

Purple dotted lines in the Fig. 4 show the $\langle\sigma v\rangle_{\gamma \gamma}$ and $\tau_{\gamma \nu}$ constraints for various DM density profiles without the systematic uncertainties. The cuspy profiles show better constraints since they have larger $J$-factors and better background-to-noise ratios. Because of smaller energy dispersion and low statistics of DAMPE data, more fluctuations appear in the constraints.

\section{Summary}

DAMPE has an excellent energy resolution due to its thick BGO calorimeter, and therefore has an advantage in detecting sharp structures. In this work, we use 5.0 years of DAMPE data to search for spectral lines from $10 \mathrm{GeV}$ to $300 \mathrm{GeV}$. To improve the sensitivity to line signals, two types of gamma-ray data sets, the LineSearch and BgoOnly data sets, are developed. We also make two ROIs optimized for DM density profiles for signals originating from the DM annihilation or decay in the Galaxy. We use the summed unbinned likelihood function to combine two data sets and the sliding windows technique to reduce the uncertainty from the spectral shape of background emission.

No line signals or candidates with TS value $\geq 9$ are detected, including those suggested at $133 \mathrm{GeV}$ and $43 \mathrm{GeV}$. We therefore present the $95 \%$ confidence level constraints on the annihilation 

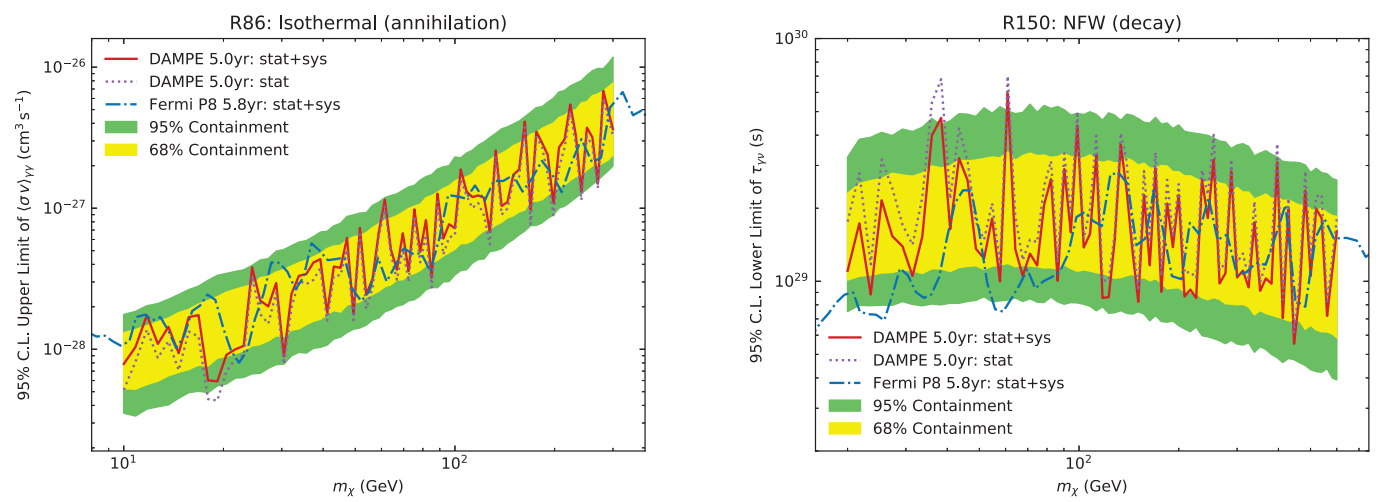

Figure 4: The 95\% confidence level constraints for different DM density profiles. The left panel show the $\langle\sigma v\rangle_{\gamma \gamma}$ upper limits of annihilating DM assuming the Isothermal profile. The right panel presents the $\tau_{\gamma \nu}$ lower limit of decaying DM assuming the NFW profile. Yellow (green) bands show the 68\% (95\%) expected containment obtained from 1000 simulations of background emission with systematic uncertainties involved. The red solid and purple dotted lines are the results with and without the systematic uncertainties respectively. The blue dot-dashed lines show the 5.8-year Fermi-LAT constraints [15].

cross section or decay lifetime with systematic uncertainties included. And most our constraints are comparable to the 5.8-year results of Fermi-LAT thanks to better energy resolution and the smaller influence of the systematic uncertainty. For the decaying DM, our lower limits on the decay lifetime are stronger for DM with mass $\lesssim 100 \mathrm{GeV}$.

\section{acknowledgements}

This work is supported in part by the National Key Research and Development Program of China (2016YFA0400200), the National Natural Science Foundation of China (Nos. U1738123, U1738210, U1738138, 12003074), the Strategic Priority Research Program of the Chinese Academy of Sciences (No. XDB23040000), the Youth Innovation Promotion Association CAS, the Entrepreneurship and Innovation Program of Jiangsu Province. In Europe the activities and data analysis are supported by the Swiss National Science Foundation (SNSF), Switzerland, the National Institute for Nuclear Physics (INFN), Italy, and the European Research Council (ERC) under the European Union's Horizon 2020 research and innovation programme (No. 851103).

\section{References}

[1] N. Aghanim, Y. Akrami, M. Ashdown, J. Aumont, et al. 2020, Astron. Astrophys., 641, 561

[2] J. L. Feng, 2010, Ann. Rev. Astron. Astrophys., 48, 495

[3] T. Bringmann, X. Huang, 2012, J. Cosmol. Astropart. Phys., 07, 054

[4] A. Ibarra, S. Lopez Gehler, and M. Pato, 2012, J. Cosmol.Astropart. Phys., 07, 043

[5] E. Charles, M. Sanchez-Conde, B. Anderson, et al. 2016, Phys. Rep., 636:1-46 
[6] Z. L. Xu, K. K. Duan, Z. Q. Shen, et al. 2018, Research in Astronomy and Astrophysics., 18, 3

[7] K. K. Duan, W. Jiang, Y. F. Liang, et al. 2019, Research in Astronomy and Astrophysics., 19, 9

[8] M. Ackermann, et al. 2013, Phys. Rev. D, 88, 082002

[9] A. Albert, G. A. Gomez-Vargas, M. Grefe, et al. 2014, J. Cosmol. Astropart. Phys., 10, 023

[10] Y. Q. Zhang, J. H. Guo, Y. Liu, et al. 2019, Research in Astronomy and Astrophysics., 19, 9

[11] J. F. Navarro, C. S. Frenk, and S. D. M. White, 1996, Astrophys. J., 462, 563

[12] J. Einasto, 1965, Trudy Astrofzicheskogo Instituta Alma-Ata, 5, 87

[13] J. F. Navarro, A. Ludlow, V. Springel, J. Wang, et al. 2010, Mon. Not. R. Astron. Soc., 402, 21

[14] J. N. Bahcall, R. M. Soneira, 1980, Astrophys. J. Suppl., 44, 73

[15] M. Ackermann, M. Ajello, A. Albert, B. Anderson, W. B.Atwood, et al. 2015, Phys. Rev. D., 91,122002

[16] Y. F. Liang, Z. Q. Xia, Z. Q. Shen, et al. 2016, Phys. Rev. D, 94, 10

[17] W. Cash, 1979, Astrophys. J., 228, 939

[18] F. James and M. Roos, 1975, Comput. Phys. Commun., 10, 343

[19] C. Weniger, 2012, J. Cosmol. Astropart. Phys., 08, 007 


\section{Full Authors List: DAMPE Collaboration}

F. Alemanno ${ }^{1,2}$, Q. An ${ }^{3,4}$, P. Azzarello ${ }^{5}$, F. C. T. Barbato ${ }^{1,2}$, P. Bernardini ${ }^{6,7}$, X. J. Bi ${ }^{8,9}$, M. S. Cai ${ }^{10,11}$, E. Casilli6, ${ }^{6}$, E. Catanzani ${ }^{12}$, J. Chang ${ }^{10,11}$, D. Y. Chen ${ }^{9,10}$, J. L. Chen ${ }^{13}$, Z. F. Chen ${ }^{10,11}$, M. Y. Cui ${ }^{10}$, T. S. Cui ${ }^{14}$, Y. X. Cui ${ }^{10,11}$, H. T. Dai ${ }^{3,4}$, A. De Benedittis ${ }^{6,7}$, I. De Mitri ${ }^{1,2}$, F. de Palma ${ }^{6,7}$, M. Deliyergiyev ${ }^{5}$, M. Di Santo ${ }^{6,7}$, Q. Ding ${ }^{10,11}$, T. K. Dong ${ }^{10}$, Z. X. Dong ${ }^{14}$, G. Donvito ${ }^{15}$, D. Droz ${ }^{5}$, J. L. Duan ${ }^{13}$, K. K. Duan ${ }^{10}$, D. D’Urso ${ }^{12,1}$, R. R. Fan ${ }^{8}$, Y. Z. Fan ${ }^{10,11}$, K. Fang ${ }^{8}$, F. Fang ${ }^{13}$, C. Q. Feng ${ }^{3,4}$, L. Feng ${ }^{10}$, P. Fusco ${ }^{15,16}$, M.

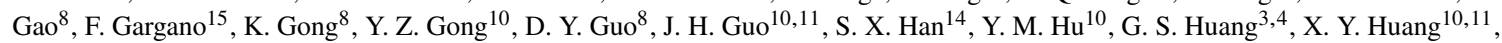
Y. Y. Huang ${ }^{10}$, M. Ionica ${ }^{12}$, W. Jiang ${ }^{10,11}$, J. Kong ${ }^{13}$, A. Kotenko ${ }^{5}$, D. Kyratzis ${ }^{1,2}$, S. J. Lei ${ }^{10}$, W. H. Li ${ }^{10,11}$, W. L. Li ${ }^{14}$, X. Li ${ }^{10}$, X. Q.

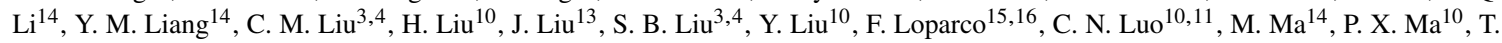
$\mathrm{Ma}^{10}$, X. Y. Ma ${ }^{14}$, G. Marsella ${ }^{6,7,2}$, M. N. Mazziotta ${ }^{15}$, D. Mo ${ }^{13}$, X. Y. Niu ${ }^{13}$, X. Pan ${ }^{10,11}$, A. Parenti ${ }^{1,2}$, W. X. Peng ${ }^{8}$, X. Y. Peng ${ }^{10}$, C. Perrina ${ }^{5,3}$, R. Qiao ${ }^{8}$, J. N. Rao ${ }^{14}$, A. Ruina ${ }^{5}$, M. M. Salinas ${ }^{5}$, G. Z. Shang ${ }^{14}$, W. H. Shen ${ }^{14}$, Z. Q. Shen ${ }^{10}$, Z. T. Shen ${ }^{3,4}$, L. Silveri ${ }^{1,2}$, J. X. Song ${ }^{14}$, M. Stolpovskiy ${ }^{5}$, H. Su ${ }^{13}$, M. Su${ }^{17}$, H. R. Sun ${ }^{3,4}$, Z. Y. Sun ${ }^{13}$, A. Surdo ${ }^{7}$, X. J. Teng ${ }^{14}$, A. Tykhonov ${ }^{5}$, H. Wang ${ }^{14}$, J. Z. Wang ${ }^{8}$, L. G. Wang ${ }^{14}$, S. Wang ${ }^{10,11}$, S. X. Wang ${ }^{10,11}$, X. L. Wang ${ }^{3,4}$, Y. Wang ${ }^{3,4}$, Y. F. Wang ${ }^{3,4}$, Y. Z. Wang ${ }^{10}$, D. M. Wei ${ }^{10,11}$, J. J. Wei ${ }^{10}$, Y. F. $\mathrm{Wei}^{3,4}$, D. $\mathrm{Wu}^{8}$, J. Wu ${ }^{10,11}$, L. B. Wu ${ }^{1,2}$, S. S. Wu ${ }^{14}$, X. Wu ${ }^{5}$, Z. Q. Xia ${ }^{10}$, E. H. Xu ${ }^{3,4}$, H. T. Xu ${ }^{14}$, Z. H. Xu ${ }^{10,11}$, Z. L. Xu ${ }^{10}$, Z. Z. Xu ${ }^{3,4}$, G. F. Xue ${ }^{14}$, H. B. Yang ${ }^{13}$, P. Yang ${ }^{13}$, Y. Q. Yang ${ }^{13}$, H. J. Yao ${ }^{13}$, Y. H. Yu ${ }^{13}$, G. W. Yuan ${ }^{10,11}$, Q. Yuan ${ }^{10,11}$, C. Yue ${ }^{10}$, J. J. Zang ${ }^{10,4}$, F. Zhang ${ }^{8}$, S. X. Zhang ${ }^{13}$, W. Z. Zhang ${ }^{14}$, Y. Zhang ${ }^{10}$, Y. J. Zhang ${ }^{13}$, Y. L. Zhang ${ }^{3,4}$, Y. P. Zhang ${ }^{13}$, Y. Q. Zhang $^{10}$, Z. Zhang ${ }^{10}$, Z. Y. Zhang ${ }^{3,4}$, C. Zhao ${ }^{3,4}$, H. Y. Zhao ${ }^{13}$, X. F. Zhao ${ }^{14}$, C. Y. Zhou ${ }^{14}$, and Y. Zhu ${ }^{14}$

${ }^{1}$ Gran Sasso Science Institute (GSSI), Via Iacobucci 2, I-67100 L'Aquila, Italy

${ }^{2}$ Istituto Nazionale di Fisica Nucleare (INFN) -Laboratori Nazionali del Gran Sasso, I-67100 Assergi, L'Aquila, Italy

${ }^{3}$ State Key Laboratory of Particle Detection and Electronics, University of Science and Technology of China, Hefei 230026, China

${ }^{4}$ Department of Modern Physics, University of Science and Technology of China, Hefei 230026, China

${ }^{5}$ Department of Nuclear and Particle Physics, University of Geneva, CH-1211, Switzerland

${ }^{6}$ Dipartimento di Matematica e Fisica E. De Giorgi, Universita' del Salento, I-73100, Lecce, Italy

${ }^{7}$ Istituto Nazionale di Fisica Nucleare (INFN) - Sezione di Lecce, I-73100, Lecce, Italy

${ }^{8}$ Institute of High Energy Physics, Chinese Academy of Sciences, Yuquan Road 19B, Beijing 100049, China

${ }^{9}$ University of Chinese Academy of Sciences, Yuquan Road 19A, Beijing 100049, China

${ }^{10}$ Key Laboratory of Dark Matter and Space Astronomy, Purple Mountain Observatory, Chinese Academy of Sciences, Nanjing 210023 , China

${ }^{11}$ School of Astronomy and Space Science, University of Science and Technology of China, Hefei 230026, China

${ }^{12}$ Istituto Nazionale di Fisica Nucleare (INFN) - Sezione di Perugia, I-06123 Perugia, Italy

${ }^{13}$ Institute of Modern Physics, Chinese Academy of Sciences, Nanchang Road 509, Lanzhou 730000, China

${ }^{14}$ National Space Science Center, Chinese Academy of Sciences, Nanertiao 1, Zhongguancun, Haidian district, Beijing 100190, China

${ }^{15}$ Istituto Nazionale di Fisica Nucleare (INFN) - Sezione di Bari, I-70125, Bari, Italy

${ }^{16}$ Dipartimento di Fisica "M. Merlin" dell'Universita' e del Politecnico di Bari, I-70126, Bari, Italy

${ }^{17}$ Department of Physics and Laboratory for Space Research, the University of Hong Kong, Pok Fu Lam, Hong Kong SAR, China

${ }^{1}$ Now at Universita‘ di Sassari, Dipartimento di Chimica e Farmacia, I-07100, Sassari, Italy.

${ }^{2}$ Now at Universita“ degli Studi di Palermo, Dipartimento di Fisica e Chimica "E. Segre"”, via delle Scienze ed. 17, I-90128 Palermo, Italy.

${ }^{3}$ Also at Institute of Physics, Ecole Polytechnique Federale de Lausanne (EPFL), CH-1015 Lausanne, Switzerland.

${ }^{4}$ Also at School of Physics and Electronic Engineering, Linyi University, Linyi 276000, China. 